\title{
How to sequence treatment in relapsed ovarian cancer
}

\author{
Sandro Pignata*,1 \& Sabrina Chiara Cecere ${ }^{1}$ \\ ${ }^{1}$ Department of Urology \& Gynecology, Istituto Nazionale Tumori IRCCS Fondazione G. Pascale, Naples, Italy \\ *Author for correspondence: s.pignata@istitutotumori.na.it
}

\begin{abstract}
Numerous disease- and patient-related factors must be considered when selecting systemic therapy for recurrent ovarian cancer. Anti-angiogenics (bevacizumab) and poly(ADP-ribose) polymerase inhibitors (olaparib, niraparib and rucaparib) have an important role as maintenance of platinum-based chemotherapy for recurrent disease and their use in the first-line setting of advanced-stage disease is becoming established. As previous exposure to none, one or both of these drug classes is integral to selecting next therapy, front-line use impacts on options available to treat recurrent disease. A key strategy to delay platinum resistance and improve prognosis of recurrent disease is to alternate treatments with different mechanisms of action. The multiple mechanisms of trabectedin and its complementarity with platinum allow intercalation between platinum regimens in potentially platinum-responsive patients with recurrent disease.
\end{abstract}

First draft submitted: 5 November 2020; Accepted for publication: 7 December 2020; Published online: 23 December 2020

Keywords: antiangiogenics • ovarian cancer • PARP inhibitors • platinum-based chemotherapy • recurrent - trabectedin

\section{Current scenario of ovarian cancer recurrence}

In recent years, several agents used to treat recurrent ovarian cancer have been escalated to front-line therapy, necessitating modifications to treatment algorithms used to guide daily practice decisions. The newer approach to ovarian cancer involves selecting the most appropriate first-line treatment, then designing an optimal treatment sequence to manage recurrent disease given that most patients relapse despite optimal surgical cytoreduction and appropriate first-line chemotherapy [1].

Historically, the main driver of therapeutic decision-making in recurrent ovarian cancer was the platinumfree interval (PFI) which is based on time to progression after platinum treatment. Patient subsets were defined according to PFI duration: refractory (progression during treatment), resistant ( $<6$ months), partially sensitive (6-12 months) or fully sensitive (>12 months) [2]. Within each category, patients were treated similarly, without consideration of individual characteristics. However, resistance to platinum-based treatment is not a categorical variable. Evidence is clear that additional patient- and disease-related factors must be considered when selecting the best treatment for recurrent disease (Table 1) [3]. This concept has been fully integrated into the treatment algorithm for recurrent disease featured in the current European Society for Medical Oncology (ESMO)/European Society of Gynaecological Oncology (ESGO) consensus guidelines for ovarian cancer. Two categories of patients with recurrent disease are defined: those responsive to platinum for whom platinum-based therapy might be the best option; those with early symptomatic relapse, progression on prior platinum or platinum intolerability for whom platinum therapy might not be the best option [3]. This review focuses on treatment sequencing in patients with potentially platinum-responsive recurrent disease.

As elaborated elsewhere in the review, the move toward incorporating anti-angiogenic agents (bevacizumab) and poly(ADP-ribose) polymerase inhibitors (PARPi; olaparib, niraparib, rucaparib) into the first-line treatment of ovarian cancer has important implications for recurrent disease, as previous exposure to none, one or both of these drug classes influences treatment selection. 
Table 1. Factors to consider when selecting treatment for patients with recurrent ovarian cancer.

Tumor histology/biology

Number of previous lines of therapy

Prior response to treatment

Treatment-free interval for platinum

Persistent toxicity

Presence of symptoms

Patient preference

Data taken from [3].

\section{Importance of tumor biology on treatment selection}

Tumor biology is a key parameter for treatment selection in recurrent ovarian cancer. About $50 \%$ of patients with high-grade serous ovarian cancers have homologous recombination deficiencies (HRD) such as germline or somatic BRCA1/2 mutations which exhibit specific clinical behaviors and improved response to platinum-based chemotherapy and PARPi [4]. The HRD mechanism in ovarian cancer is associated with a higher neoantigen load, which makes the tumor more immunogenic and potentially more suitable than other tumor types for combined treatments (e.g., checkpoint inhibitors and PARPi) [5]. In homologous recombination proficient patients, tumor cell growth and proliferation are due to abnormalities such as amplification of the cell cycle dependent gene CCNE1 (cyclin E1) or loss of PTEN, RB1 or NF1 genes [6].

As reviewed by Kim and colleagues, tumor cells are composed of different clones defined as cells sets that share a common genotype [7]. The interaction of cancer cells with the microenvironment modulates tumor heterogeneity, which affects treatment response. Intratumor heterogeneity has been implicated as the main cause of treatment failure by chemoresistance in many cancer types including ovarian cancer. Intratumor heterogeneity is a result of evolutionary forces of selection pressures which increase a tumor's ability to adapt to an incompatible microenvironment. Tumor evolution models include linear, branching, neutral and punctuated, with varying implications for the diagnosis, prognosis and treatment of cancer patients. The degree of intratumor heterogeneity in ovarian cancer patients is known to correlate with the recurrence rate. Initial heterogeneity develops further during disease progression. Studies of high-grade serous ovarian cancer consistently show a dynamic entity composed of multiple populations of genetically and phenotypically distinct subclones evolved from a single ancestral clone, with patient-specific patterns of branched evolution. Medical therapies used to treat this subtype exert a selective pressure that supports the survival and proliferation of platinum-resistant cell clones [7].

A major goal of treating ovarian cancer is to delay the emergence of platinum resistance for as long as possible. Key strategies to delay resistance are to: alternate chemotherapeutic regimens using agents with different mechanisms of action; adapt the choice of agents to the biological characteristics of the tumor; and alternate short-term pressure (i.e., chemotherapy) with continuous pressure (i.e., maintenance therapy) [8]. A hope for future is to be able to select next treatment based on a biological assay.

\section{Importance of previous therapy on treatment selection}

Classes of biological therapy currently indicated to treat recurrent ovarian cancer, and recommended as possible maintenance therapy for patients with potentially platinum-responsive disease, are anti-angiogenesis agents (bevacizumab) and PARPi (olaparib, niraparib and rucaparib) [3]. In selecting next therapy for recurrent disease, four clinical scenarios can be defined based on patients' previous exposure to biological therapy:

- Scenario 1: chemotherapy only; no previous bevacizumab, no previous PARPi;

- Scenario 2: chemotherapy plus PARPi, no previous bevacizumab;

- Scenario 3: chemotherapy plus bevacizumab, no previous PARPi;

- Scenario 4: chemotherapy plus bevacizumab plus PARPi.

\section{Scenario 1}

In patients potentially responsive to platinum with no previous exposure to biological therapy, ESMO-ESGO guidelines recommend platinum-based rechallenge plus bevacizumab for those with a high disease burden and priority for a symptomatic response [3]. The preferred option for remaining patients is platinum-based rechallenge followed by a PARPi (Figure 1) [3]. PARPi maintenance therapy has been shown to be highly effective in prolonging progression-free survival (PFS), especially in patients with germline BRCA mutations or HRD tumors [9-11]. 


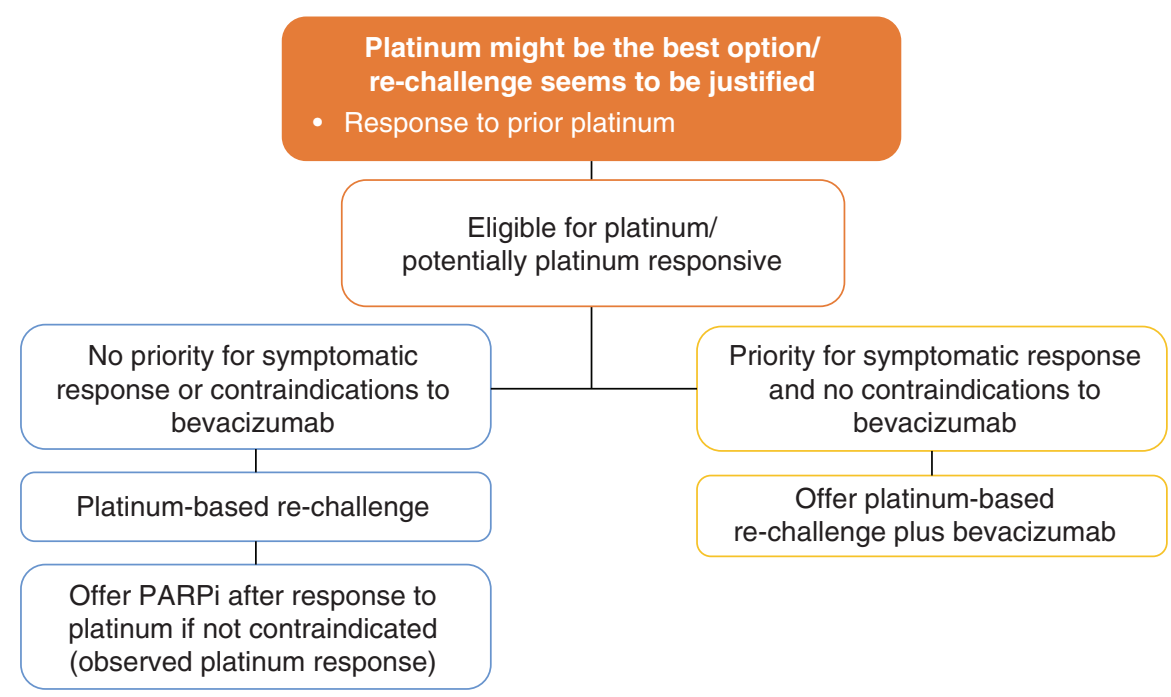

Figure 1. European Society of Gynaecological Oncology/European Society for Medical Oncology consensus guideline recommendations for recurrent ovarian cancer patients previously exposed to chemotherapy in whom platinum rechallenge might be the best option.

ESGO: European Society of Gynaecological Oncology; ESMO: European Society for Medical Oncology; PARPi: Poly(ADP-ribose) polymerase inhibitors.

Reproduced with permission from [3].

\section{Scenario 2}

In potentially platinum-responsive patients with previous exposure to a PARPi, ESMO-ESGO guidelines recommend platinum-based rechallenge plus bevacizumab [3]. In the OCEANS study of patients with platinum-sensitive recurrent ovarian cancer, carboplatin and gemcitabine plus bevacizumab followed by bevacizumab until progression significantly improved PFS compared with carboplatin and gemcitabine alone (12.4 vs 8.4 months; hazard ratio [HR]: 0.48; 95\% CI: 0.39-0.61; p < 0.0001) [12]. Another option is carboplatin and pegylated liposomal doxorubicin (PLD) plus bevacizumab which was recently shown to be superior to carboplatin and gemcitabine plus bevacizumab in improving median PFS (13.3 vs 11.7 months; HR: 0.80; 95\% CI: 0.68-0.96; $p=0.0128$ ) in patients with recurrent disease [13].

\section{Scenario 3}

In potentially platinum-responsive patients previously exposed to bevacizumab, platinum-based rechallenge followed by PARPi maintenance therapy is effective irrespective of $B R C A$ mutation and HRD status (Table 2) [10,14]. Olaparib [15], niraparib [16] and rucaparib [17] can also be considered for use as monotherapy in patients with recurrent disease who have received several previous lines of treatment [3]. However, there is some question as to whether patients who progress on PARPi are sensitive to further chemotherapy. Although results of the Phase III olaparib [9] and niraparib [10] trials reported superiority of PARPi maintenance over placebo for median time to second progression (PFS2), a retrospective study from Italy found that overall response rates were disappointing in platinum-sensitive patients with $B R C A$ mutation who received chemotherapy after olaparib progression $(\mathrm{n}=66$ evaluable for response). Response rates of 22.2, 11.1 and $9.5 \%$ in patients with a PFI of $>12,6-12$ and $<6$ months, respectively, suggested crossresistance [18]. Considering that PARPi maintenance therapy may represent a new standard in the first-line setting, these findings may have implications in terms of selecting treatment for recurrent disease, although this remains to be confirmed in large prospective well-controlled studies. At the molecular level, resistance mechanisms described in ovarian cancer patients with relapse during PARPi treatment include secondary mutations in BRCA1 or BRCA2 genes; loss of $\mathrm{p} 53$-binding protein 1 function; and overexpression of the membrane-bound drug efflux transporter (glycoprotein-P/multidrug resistance protein 1 ) with consequent efflux of PARPi [19]. Further insight is required to fully understand the processes. Importantly, resistance to PARPi in ovarian cancer metastases is a heterogeneous phenomenon [20] that is not present in other metastatic lesions which offer the possibility of surgical intervention to remove resistant disease. 
Table 2. Median progression-free survival observed with PARPi maintenance therapy in platinum-sensitive ovarian cancer patients in response to second-line platinum-based chemotherapy according to the presence or absence of germline BRCA mutations and homologous recombination deficiency status.

\begin{tabular}{|c|c|c|}
\hline Patient subgroup & Niraparib vs placebo & Olaparib \\
\hline gBRCAmut & $\begin{array}{l}n=203 \\
21.0 \text { vs } 5.5 \text { months } \\
\text { HR: } 0.27 ; p<0.001\end{array}$ & $\begin{array}{l}\mathrm{n}=75 \\
11.0 \text { months } \\
95 \% \mathrm{Cl}: 8.3-12.2\end{array}$ \\
\hline Non-gBRCAmut & $\begin{array}{l}n=350 \\
9.3 \text { vs } 3.9 \text { months } \\
\text { HR: } 0.45 ; p<0.001\end{array}$ & $\begin{array}{l}\mathrm{n}=25 \\
10.8 \text { months } \\
95 \% \mathrm{Cl}: \text { 7.3-NE }\end{array}$ \\
\hline Non-gBRCAmut HRD-pos & $\begin{array}{l}n=162 \\
12.9 \text { vs } 3.8 \text { months } \\
\text { HR: } 0.38 ; \mathrm{p}<0.001\end{array}$ & $\begin{array}{l}\mathrm{n}=68 \\
7.2 \text { months } \\
95 \% \mathrm{Cl}: 5.3-7.6\end{array}$ \\
\hline HRD-neg & $\begin{array}{l}\mathrm{n}=134 \\
6.9 \text { vs } 3.8 \text { months } \\
\text { HR: } 0.58 ; \mathrm{p}<0.001\end{array}$ & $\begin{array}{l}\mathrm{n}=89 \\
5.4 \text { months } \\
95 \% \mathrm{Cl}: 3.7-5.6\end{array}$ \\
\hline
\end{tabular}

\section{Scenario 4}

In the Phase III PAOLA-1/ENGOT-OV25 trial of patients with newly-diagnosed ovarian cancer, the addition of olaparib maintenance therapy to standard first-line platinum-taxane chemotherapy plus bevacizumab provided a significant median PFS benefit compared with placebo (22.1 vs 16.6 months; HR: 0.59; 95\% CI: 0.49-0.72; $\mathrm{p}<0.001$ [21], supporting its front-line use in advanced-stage disease. However, second-line options for patients who relapse after exposure to both agents are likely to be limited to platinum- or nonplatinum-based regimens without additional maintenance therapy. As this scenario is relatively recent, evidence is currently insufficient to recommend a general approach to managing recurrent disease in patients treated with the PAOLA-1/ENGOTOV25 regimen.

Although evidence to support the use of front-line PARPi maintenance therapy in patients with $B R C A$-associated tumors is strong, the influence of HRD status on optimal timing of PARPi therapy is less well defined [22,23]. Advances in HRD testing may eventually facilitate the identification of candidates for front-line PARPi such that treatment can be limited to patients most likely to benefit, and inform decisions to use platinum- or nonplatinumbased regimens given the greater sensitivity of HRD-positive tumors to platinum and PARPi [23]. Additional sequencing studies are required to address the many uncertainties faced by oncologists in a rapidly-evolving treatment environment [22,23].

\section{Importance of intercalating different mechanisms of action}

The main objective when treating recurrent ovarian cancer is to extend survival across multiple lines of therapy, using sequential treatment to delay disease progression after each new relapse, while preserving quality of life [24]. The superior prognoses observed in patients with platinum-sensitive versus platinum-resistant disease emphasizes the importance of maintaining platinum sensitivity when planning treatment lines. Although most treatment regimens for platinum-sensitive patients involve platinum, a loss of efficacy and increasing toxicity after each platinum exposure are well-known phenomena and underlie the importance of using chemotherapeutic agents with differing mechanisms of action [25]. Trabectedin plus PLD is currently the only nonplatinum combination approved to treat recurrence in patients with platinum-sensitive disease [3]. In a randomized Phase III study in platinum-sensitive patients who had relapsed after one line of platinum-based chemotherapy, the efficacy of trabectedin plus PLD was superior to that of PLD monotherapy with no impairment to quality of life [26,27]. In a prospective observational 'real-life' study of heavily pretreated patients ( $\mathrm{n}=158$ evaluable) with platinum-sensitive ovarian cancer who were treated with trabectedin plus PLD, the overall response rate was 38\%, the disease control rate was $66.5 \%$ and median PFS was 11.4 months. The combination was effective whether administered in the second $(\sim 25 \%$ of patients), third $(\sim 34 \%)$ or fourth and later $(\sim 40 \%)$ line of treatment [28].

The benefits observed with trabectedin in combination with PLD between platinum lines may derive from its multitasking mechanism of action and complementarity with platinum. Trabectedin impairs DNA repair, inhibits 
transcription, induces changes in the tumor microenvironment [29], and may restore platinum sensitivity by selecting platinum-responsive cancer cells [8].

\section{Importance of rechallenge}

Because ovarian cancer management generally involves multiple lines of treatment, rechallenge with previously administered agents plays an important role. There are studies and case reports of retreatment with platinum, paclitaxel, bevacizumab and more recently, PLD. A multicenter retrospective study evaluating PLD rechallenge in 27 patients with recurrent ovarian cancer reported a response rate of $49 \%$ (complete response 19\%, partial response $30 \%$ ) and no safety issues [30]. Retreatment with PARPi may also be an option for recurrent ovarian cancer. An ongoing Phase III clinical trial is investigating the efficacy and safety of olaparib maintenance therapy in patients with disease progression following maintenance therapy with any previous PARPi (ClinicalTrials.gov Identifier: NCT03106987) [31].

\section{Conclusion \& future perspective}

In recent years, there has been a revolution in the approach to ovarian cancer, including escalation of PARPi to front-line management of patients with newly-diagnosed advanced disease. Although the move to improve outcomes in this underserved patient population is welcome, it has raised important questions about treatment selection: Should all patients with ovarian cancer receive chemotherapy plus PARPi? Should patients with HRD and a germline BRCA mutation receive chemotherapy and PARPi plus bevacizumab? Data on the use of chemotherapy and immunotherapy plus bevacizumab are due to be released in upcoming months and, in future, insight is expected into the use of chemotherapy and PARPi plus immunotherapy. A major challenge clinicians are certain to face is to agree on criteria to select optimal first-line treatment among an increasing number of options.

As ovarian cancer patients generally receive multiple consecutive lines of therapy, forward planning is essential. Beyond PFI, multiple patient- and disease-related factors must be considered when selecting next-line therapy in relapsing patients. Identifying strategies to delay treatment resistance is an underexplored area of research and a key strategy toward improving patient prognoses. To this end, alternating chemotherapy regimens with differing mechanisms of actions appears to be a useful approach that warrants investigation in treatment sequence trials. Rechallenge is an established practice with several front-line agents for ovarian cancer. Whether rechallenge with PARPi is also possible is currently under investigation and the results are awaited with interest.

Financial \& competing interests disclosure

S Pignata: has received honoraria from AstraZeneca, Clovis, MSD, PharmaMar, Roche and Tesaro; has received research funding from AstraZeneca, MSD, Pfizer and Roche; SC Cecere: has received honoraria from AstraZeneca, PharmaMar and Tesaro. The authors have no other relevant affiliations or financial involvement with any organization or entity with a financial interest in or financial conflict with the subject matter or materials discussed in the manuscript apart from those disclosed.

Editorial assistance was provided by R Furlong and K Dechant on behalf of Content Ed Net (Madrid, Spain) with funding from PharmaMar (Madrid, Spain).

\section{Open access}

This work is licensed under the Attribution-NonCommercial-NoDerivatives 4.0 Unported License. To view a copy of this license, visit http://creativecommons.org/licenses/by-nc-nd/4.0/ 
Executive summary

Current scenario of ovarian cancer recurrence

- Escalation to front-line therapy of drugs used to treat recurrent ovarian cancer has implications as previous exposure to biological agents influences available options for recurrent disease.

- This review focuses on treatment sequencing in potentially platinum-responsive patients with recurrent ovarian cancer.

Importance of tumor biology on treatment selection

- Tumor biology has a key role in treatment selection for recurrent ovarian cancer.

- Homologous recombination deficiencies such as germline or somatic BRCA1/2 mutations exhibit specific clinical behaviors and improved response to platinum-based chemotherapy and poly(ADP-ribose) polymerase inhibitors (PARPi).

- Intratumor heterogeneity, arising from varying patterns of clonal evolution, is the main cause of treatment failure by chemoresistance and has implications for the diagnosis, prognosis and treatment of ovarian cancer patients.

- Delaying the emergence of platinum resistance for as long as possible is a major goal of ovarian cancer management.

Importance of previous therapy on treatment selection

- In potentially platinum-responsive patients with recurrent disease and no previous exposure to biological therapy, platinum-based rechallenge plus bevacizumab is recommended for those with a high disease burden and priority for a symptomatic response. Platinum-based rechallenge followed by a PARPi is recommended in remaining patients.

- In potentially platinum-responsive patients with recurrent disease and previous exposure to a PARPi, platinum-based rechallenge plus bevacizumab is recommended.

- In potentially platinum-responsive patients with recurrent disease and previous exposure to bevacizumab, platinum-based rechallenge followed by PARPi maintenance therapy is effective. PARPi monotherapy can be considered for heavily pretreated patients.

- Options for patients who relapse after first-line exposure to bevacizumab and PARPi (olaparib) are likely to be limited to platinum- or nonplatinum-based regimens without maintenance therapy.

Importance of intercalating different mechanisms of action

- Loss of efficacy and increasing toxicity after each platinum exposure underlie the importance of using chemotherapeutic agents with differing mechanisms of action.

- Trabectedin plus pegylated liposomal doxorubicin is currently the only nonplatinum combination approved to treat recurrence in patients with platinum-sensitive disease.

Importance of rechallenge

- As ovarian cancer management generally involves multiple lines of treatment, rechallenge with previously administered agents plays an important role.

- Evidence of retreatment is available for platinum, paclitaxel, bevacizumab and pegylated liposomal doxorubicin. Data on re-exposure to PARPi are not yet available.

Conclusion

- Escalation of PARPi to front-line management of patients with newly-diagnosed advanced disease has raised several questions about treatment sequencing. Forward planning is essential.

- Identifying strategies to delay treatment resistance in ovarian cancer is an underexplored area of research and a key strategy toward improving patient prognoses.

\section{References}

Papers of special note have been highlighted as: $\bullet$ of interest

1. Pignata S, Cecere SC, Du Bois A, Harter P, Heitz F. Treatment of recurrent ovarian cancer. Ann. Oncol. 28(Suppl. 8), viii51-viii56 (2017).

2. Wilson MK, Pujade-Lauraine E, Aoki D et al. Fifth Ovarian Cancer Consensus Conference of the Gynecologic Cancer InterGroup: recurrent disease. Ann. Oncol. 28, 727-732 (2017).

3. Colombo N, Sessa C, du Bois A et al. ESMO-ESGO consensus conference recommendations on ovarian cancer: pathology and molecular biology, early and advanced stages, borderline tumors and recurrent disease. Ann. Oncol. 30, 672-705 (2019).

- European Society for Medical Oncology-European Society of Gynaecological Oncology consensus conference recommendations for ovarian cancer based on best available evidence and expert agreement from a multidisciplinary panel of 40 leading experts.

4. Rodrigues da Cunha Colombo Bonadio R, Nogueira Fogace R, Costa Miranda V, Del Pilar Estevez Diz M. Homologous recombination deficiency in ovarian cancer: a review of its epidemiology and management. Clinics (Sao Paulo) 73(Suppl. 1), e450s (2018).

5. Strickland KC, Howitt BE, Shukla SA et al. Association and prognostic significance of BRCA1/2-mutation status with neoantigen load, number of tumor-infiltrating lymphocytes and expression of PD-1/PD-L1 in high grade serous ovarian cancer. Oncotarget 7(12), 13587-13598 (2016). 
6. Cancer Genome Atlas Research. Integrated genomic analyses of ovarian carcinoma. Nature 474, 609-615 (2011).

7. Kim S, Han Y, Kim SI, Kim HS, Kim SJ, Song YS. Tumor evolution and chemoresistance in ovarian cancer. NPJ Precis. Oncol. 2, 20 (2018).

- Examines strategies to overcome chemoresistance in ovarian cancer with a focus on those targeting tumoral evolution and intratumor heterogeneity.

8. Tomao F, D'Incalci M, Biagioli E, Peccatori FA, Colombo N. Restoring platinum sensitivity in recurrent ovarian cancer by extending the platinum-free interval: myth or reality? Cancer 123, 3450-3459 (2017).

9. Pujade-Lauraine E, Ledermann JA, Selle F et al. Olaparib tablets as maintenance therapy in patients with platinum-sensitive, relapsed ovarian cancer and a BRCA1/2 mutation (SOLO2/ENGOT-Ov21): a double-blind, randomised, placebo-controlled, Phase III trial. Lancet Oncol. 18, 1274-1284 (2017) (Erratum in: Lancet Oncol. 18(9), e510 (2017).

10. Mirza MR, Monk BJ, Herrstedt J et al. Niraparib maintenance therapy in platinum-sensitive, recurrent ovarian cancer. N. Engl. J. Med. 375, 2154-2164 (2016).

11. Coleman RL, Oza AM, Lorusso D et al. Rucaparib maintenance treatment for recurrent ovarian carcinoma after response to platinum therapy (ARIEL3): a randomised, double-blind, placebo-controlled, Phase III trial. Lancet 390, 1949-1961 (2017).

12. Aghajanian C, Blank SV, Goff BA et al. OCEANS: a randomized, double-blind, placebo-controlled Phase III trial of chemotherapy with or without bevacizumab in patients with platinum-sensitive recurrent epithelial ovarian, primary peritoneal, or fallopian tube cancer. $J$. Clin. Oncol. 30, 2039-2045 (2012).

13. Pfisterer J, Shannon CM, Baumann K et al. Bevacizumab and platinum-based combinations for recurrent ovarian cancer: a randomised, open-label, Phase III trial. Lancet Oncol. 21(5), 699-709 (2020).

14. Cadoo KA, Simpkins F, Mathews CA, Kabil N, Bennett J, Aghajanian C. Olaparib treatment in patients (pts) with platinum-sensitive relapsed (PSR) ovarian cancer (OC) by BRCA mutation (BRCAm) and homologous recombination deficiency (HRD) status: Phase II LIGHT study. J. Clin. Oncol. 38 (Suppl. 15), 6013-6013 (2020).

15. Penson RT, Valencia RV, Cibula D et al. Olaparib versus nonplatinum chemotherapy in patients with platinum-sensitive relapsed ovarian cancer and a germline BRCA1/2 mutation (SOLO3): a randomized Phase III trial. J. Clin. Oncol. 38(11), 1164-1174 (2020).

16. Moore KN, Secord AA, Geller MA et al. Niraparib monotherapy for late-line treatment of ovarian cancer (QUADRA): a multicentre, open-label, single-arm, Phase II trial. Lancet Oncol. 20(5), 636-648 (2019) (published correction appears inLancet Oncol. 20(5), e242 [2019]).

17. Swisher EM, Lin KK, Oza AM et al. Rucaparib in relapsed, platinum-sensitive high-grade ovarian carcinoma (ARIEL2 part 1): an international, multicentre, open-label, Phase II trial. Lancet Oncol. 18(1), 75-87 (2017).

18. Cecere SC, Giannone G, Salutari V et al. Olaparib as maintenance therapy in patients with BRCA 1-2 mutated recurrent platinum sensitive ovarian cancer: real world data and post progression outcome. Gynecol. Oncol. 156(1), 38-44 (2020).

19. Incorvaia L, Passiglia F, Rizzo S et al. "Back to a false normality": new intriguing mechanisms of resistance to PARP inhibitors. Oncotarget 8, 23891-23904 (2017).

20. Patch AM, Christie EL, Etemadmoghadam D et al. Whole-genome characterization of chemoresistant ovarian cancer. Nature 521, 489-494 (2015).

21. Ray-Coquard I, Pautier P, Pignata $S$ et al. Olaparib plus bevacizumab as first-line maintenance in ovarian cancer. N. Engl. J. Med. 381(25), 2416-2428 (2019).

- Adding maintenance olaparib to first-line standard therapy including bevacizumab in patients with advanced ovarian cancer provided a significant progression-free survival benefit, which was substantial in patients with homologous recombination deficiencies-positive tumors, including those without a $B R C A$ mutation.

22. Onstad M, Coleman RL, Westin SN. Movement of poly-ADP ribose (PARP) inhibition into frontline treatment of ovarian cancer. Drugs 80(15), 1525-1535 (2020).

- Review discusses the escalation of PARP inhibitor (PARPi) to front-line therapy in ovarian cancer.

23. Mirza MR, Coleman RL, González-Martín A et al. The forefront of ovarian cancer therapy: update on PARP inhibitors. Ann. Oncol. 31(9), 1148-1159 (2020).

- Review discusses the escalation of PARPi to front-line therapy in ovarian cancer.

24. DiSilvestro P, Alvarez Secord A. Maintenance treatment of recurrent ovarian cancer: is it ready for prime time? Cancer Treat. Rev. 69, 53-65 (2018).

25. Fotopoulou C. Limitations to the use of carboplatin-based therapy in advanced ovarian cancer. EJC Suppl. 12(2), 13-16 (2014).

26. Monk BJ, Herzog TJ, Kaye SB et al. Trabectedin plus pegylated liposomal doxorubicin in recurrent ovarian cancer. J. Clin. Oncol. 28(19), 3107-3114 (2010).

27. Krasner CN, Poveda A, Herzog TJ et al. Patient-reported outcomes in relapsed ovarian cancer: results from a randomized Phase III study of trabectedin with pegylated liposomal doxorubicin (PLD) versus PLD alone. Gynecol. Oncol. 127(1), 161-167 (2012). 
28. Pignata S, Scambia G, Mazzei T, Arruti Barbia M, Naglieri E, de Sande LM. Trabectedin plus pegylated liposomal doxorubicin (PLD) in patients with platinum-sensitive recurrent ovarian cancer (PSROC) regardless of prior use of antiangiogenics: first results of an observational, prospective study. Ann. Oncol. 29(Suppl. 8), viii352 (2018).

29. D'Incalci M, Galmarini CM. A review of trabectedin (ET-743): a unique mechanism of action. Mol. Cancer Ther. 9, 2157-2163 (2010).

30. Tripodi E, Cormio G, De Giorgi U et al. Pegylated liposomal doxorubicin re-challenge in patients with ovarian cancer relapse: a multicenter retrospective study. Int. J. Gynecol. Cancer 29, 153-157 (2019).

31. U.S. National Institutes of Health. A Phase IIIb, randomised, double-blind, placebo-controlled, multicentre study of olaparib maintenance retreatment in patients with epithelial ovarian cancer previously treated with a PARPi and responding to repeat platinum chemotherapy. https://clinicaltrials.gov/ct2/show/NCT03106987 